\title{
Naturmessungen an Talsperren im Zusammenspiel mit numerischen Berechnungen
}

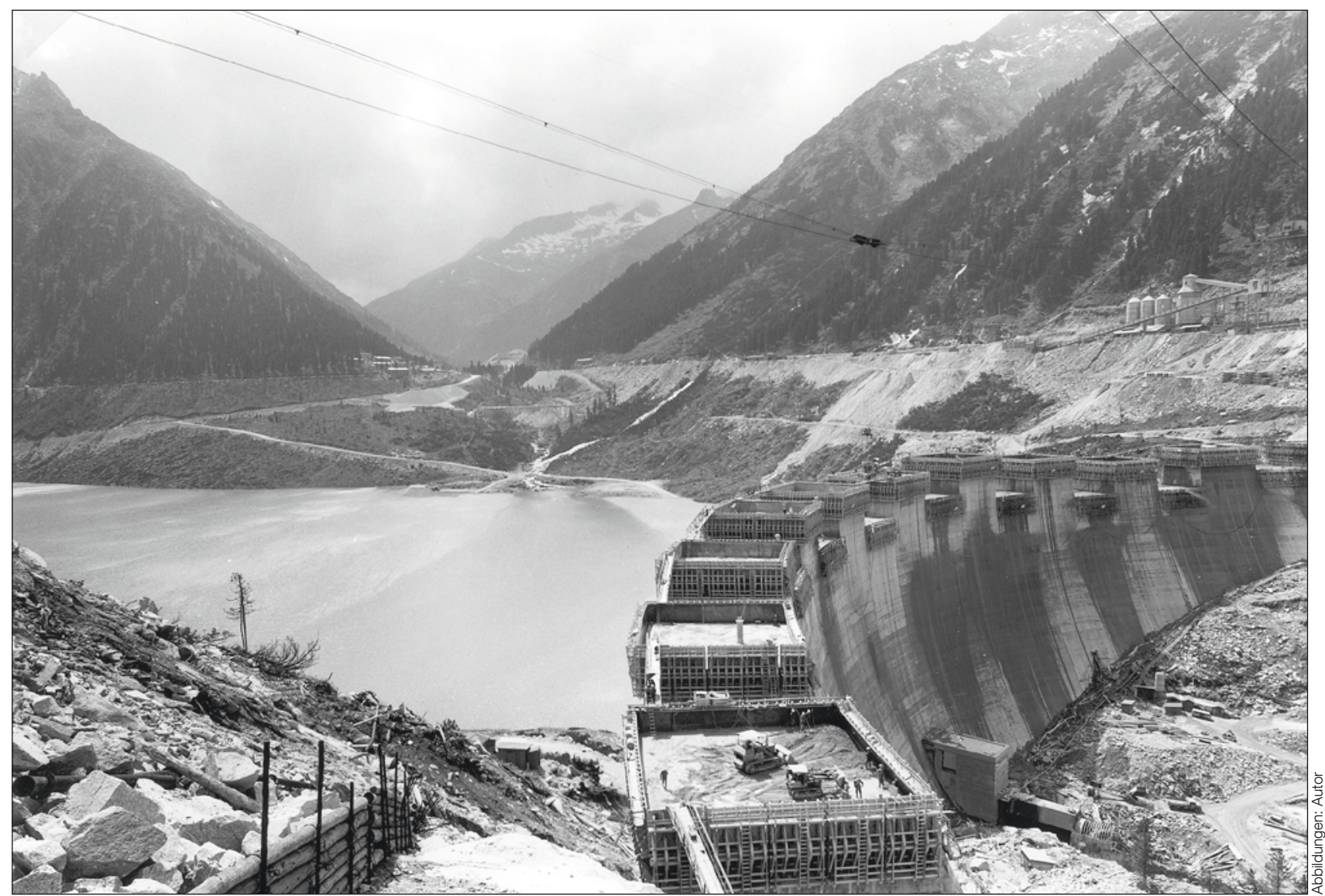

Abb. 1: Sperre Schlegeis - Bauzustand.

Zusammenfassung: Eine geeignete Wahl der Materialparameter ist für die Berechnung der Standsicherheit von Sperrenbauwerken von essentieller Bedeutung. Daten aus Messungen an bestehenden Talsperren können als Grundlage für eine Rückrechnung dieser Materialparameter an einem dafür geeigneten Modell herangezogen werden. Im vorliegenden Beitrag wird exemplarisch die Bestimmung der sogenannten dynamischen E-Moduli für Fels und Beton für die Sperre Schlegeis mithilfe von Naturmessungen und eines Finite Elemente Modells dargestellt.

Field measurements for dams in conjunction with numerical calculations

Summary: The selection of proper material parameters is essential for analysing the stability of large dam structures. Data from measurements on existing dams can be used as a basis for the back-calculation of such parameters on a suitable model. This article demonstrates, by way of example, the determination of the so-called dynamic moduli for rock and concrete for the Schlegeis dam in Tyrol by use of field measurements and a finite element model.

\section{Einleitung}

Talsperren sind im Allgemeinen in Österreich gut überwachte und instrumentierte Bauwerke. Die Überwachung dient dem sicheren und verlässlichen Betrieb der Anlagen über ihre lange Lebensdauer und stellt die Grundlage zur Beurteilung des
Langzeitverhaltens dar. Die Bauwerke und ihr Verhalten unter verschiedenen Belastungssituationen werden mit hoher Genauigkeit und meist in Echtzeit mittels Fernüberwachung beobachtet. (1)

Aus der Gesamtheit der Daten, die aus Messungen von Verformung, Temperatur, Porenwasserdruck etc. gewonnen werden, können kritische Situationen für die Integrität des Bauwerks identifiziert und damit die Vorhersagen über dessen Reaktion auf verschiedene Betriebsszenarien getroffen werden.

Neben den ständigen Überwachungseinrichtungen werden in mehr oder weniger regelmäßigen Abständen Inspektionen bzw. vereinzelt auch Versuche wie z.B. zum Betonzustand oder dem Eigenschwingverhalten der Sperren durchgeführt. 


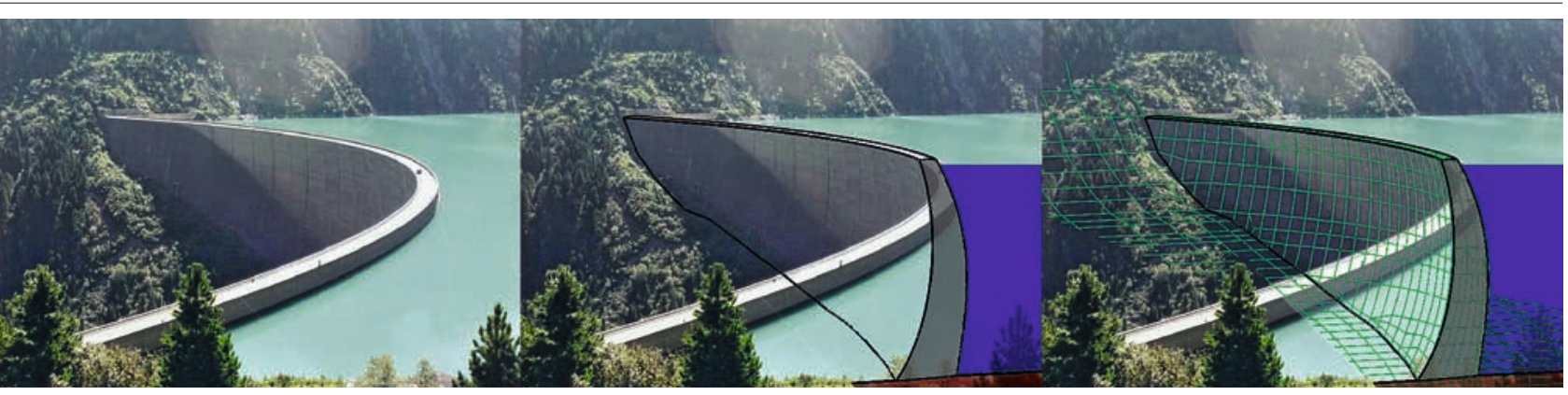

Abb. 2: Sperre Schlegeis: Natur - Geometrie - FE-Netz.

Die damit erhaltenen Informationen stellen wichtige Eingangs- bzw. Vergleichsdaten für Berechnungen dar, um die Qualität der gewählten Parameter zu verbessern und so die Aussagekraft des Berechnungsmodells der betrachteten Struktur zu erhöhen. Ebenso werden dadurch Fortschritte in der Auslegung und Modellierung für zukünftige Bauwerke durch ein besseres Verständnis der Struktur schon in der Planung und Berechnung erzielt.

Im vorliegenden Artikel wird, als Beispiel anhand einer Erdbebenberechnung für österreichische Talsperren (3), die Anwendung von Messdaten (2) zur Bestimmung von Materialparametern und Modellverhalten für eine Finite Elemente Berechnung illustriert.

\section{GEWÖLBEMAUER SCHLEGEIS}

\subsection{Allgemeines}

Die Sperre Schlegeis wurde in den Jahren 1969 bis 1971 errichtet (Abb. 1) und stellt das Absperrbauwerk des Schlegeis Speichers dar, der zur Kraftwerksgruppe Zemm/Ziller in Tirol gehört.

Bei einer Sperrendicke von $9 \mathrm{~m}$ an der Krone und einer maximalen Sperrendicke von $34 \mathrm{~m}$ wurden ca. $960000 \mathrm{~m}^{3}$ Beton verbaut. Das Bauwerk ist $131 \mathrm{~m}$ hoch und mit einer Kronenbogenlänge von $725 \mathrm{~m}$ weit gespannt. Der Speicher fasst bei einem Stauziel von $1782 \mathrm{~m}$ ü. A. (Absenkziel $1680 \mathrm{~m}$ ü. A.) rund 127 Mill. $\mathrm{m}^{3}$. (3)

\subsection{Natur und Modell}

Das Finite Elemente Modell wurde anhand der Geometriedaten ( $A b b .2)$ über die Sperre erstellt und besteht aus rund 1100 Elementen mit ca. 7000 Knoten. Die Elemente haben einen quadratischen Verschiebungsansatz. Daraus ergibt sich in etwa eine Anzahl von 20000 Freiheitsgraden.
Für das Modell wird die Geometrie des Tales vereinfacht, wobei die Aufstandslinie ober- und unterwasserseitig zu den Modellgrenzen hin projiziert wird. Der erfasste Geländeausschnitt erstreckt sich etwa $940 \mathrm{~m}$ in Tallängs- und $1200 \mathrm{~m}$ in Talquerrichtung. Eine Tiefe von $130 \mathrm{~m}$ unter der Talsohle wird abgebildet. (Abb. 3)

\subsection{Berechnung der Eigenfrequenzen}

Für die Berechnung wird ein linear elastisches Materialgesetz für den Sperrenbeton und den Felsuntergrund verwendet. Der Dichte des Sperrenbetons wird hierbei mit 2,4 to $/ \mathrm{m}^{3}$ angesetzt und der Fels als masselos betrachtet. Dem Einfluss des Reservoirs auf die Eigenfrequenz wird über die addierten Massen nach Westergaard Rechnung getragen.

Die Ermittlung der Eigenfrequenzen erfolgt für verschiedene Wasser- bzw. Füllstände des Speichers. Die Sohl- und Blockfugen werden in dieser Phase der Berechnung, nach einer Sensitivitätsanalyse, als geschlossen betrachtet.

\subsection{Eigenfrequenzen aus Messungen}

Bei den durchgeführten Messungen sinkt die ermittelte Eigenfrequenz bei nahezu leerem Speicher wieder ab. Dies deutet auf eine Öffnung der Blockfugen hin, die mit dem gewählten numerischen Modell nicht abzubilden ist. Für die Ermittlung der
Kennwerte werden somit nur die Messungen bei $7 \mathrm{~m}$ und $51 \mathrm{~m}$ unter Vollstau herangezogen (Abb. 4).

\subsection{Anpassung der Materialkennwerte}

Für die Anpassung der Berechnungsergebnisse an die gemessenen Eigenfrequenzen wird zunächst der dynamische Verformungsmodul des Sperrenbetons, ausgehend von Werten aus früheren Untersuchungen (FEM-Berechnung, 1994), variiert. $(A b b .5)$

Dabei ergeben sich für den Sperrenbeton zu hohe Werte. Deshalb wird in einem nächsten Schritt der E-Modul des Felsens angehoben. (Abb. 6)

Somit ergibt sich im Vergleich mit den Ergebnissen aus den Messungen ein EModul von $40000 \mathrm{MPa}$ für den Sperrenbeton und den Felsuntergrund. Je nach Stau-

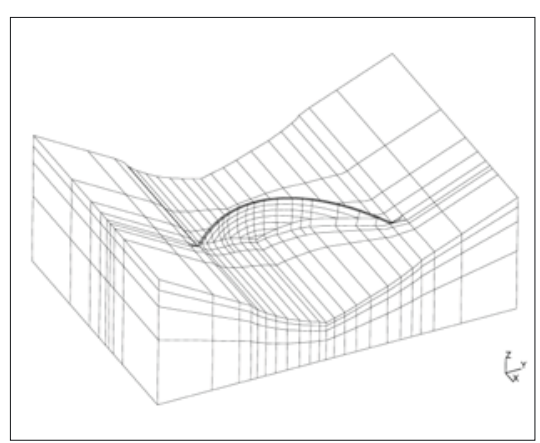

Abb. 3: Sperre Schlegeis - Finite Elemente Modell. (4)

ABBILDUNG 4

Sperre Schlegeis - Eigenfrequenzen aus Messungen

Messungen - TU Wien, Arsenal 1995/1996

\begin{tabular}{lll}
\hline Mode & Eigenfrequenz [Hz] & Eigenfrequenz [Hz] \\
& $\mathbf{7}$ unter Vollstau (1775 müA) & $\mathbf{5 1 ~}$ m unter Vollstau (1731 müA) \\
\hline 1 & $\mathbf{1 , 9 2}$ & $\mathbf{2 , 2 1}$ \\
\hline 2 & 2,05 & 2,34 \\
\hline 3 & 2,47 & 2,75 \\
\hline 4 & 2,93 & 3,23 \\
\hline 5 & 3,53 & 3,84 \\
\hline
\end{tabular}




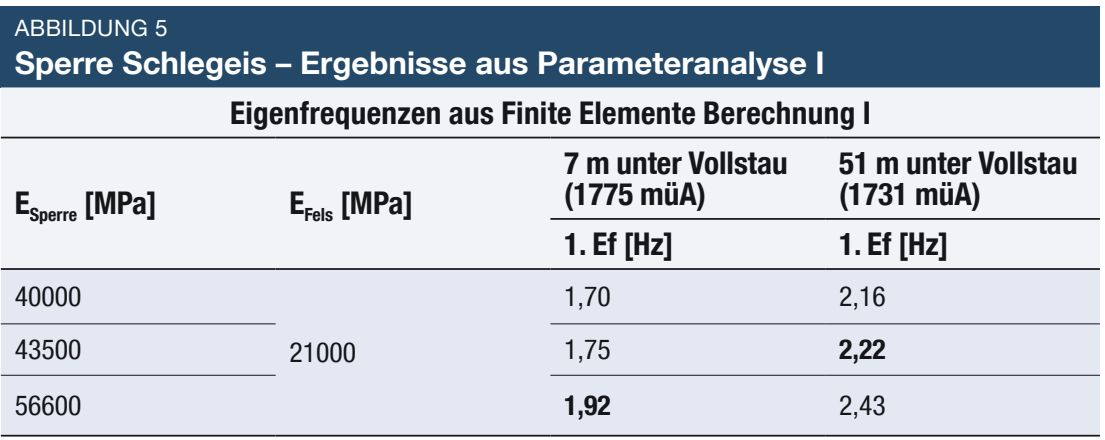

\begin{tabular}{|c|c|c|c|}
\hline \multicolumn{4}{|c|}{ Eigenfrequenzen aus Finite Elemente Berechnung II } \\
\hline \multirow{2}{*}{$\mathrm{E}_{\text {Sperre }}[\mathrm{MPa}]$} & \multirow{2}{*}{$\mathrm{E}_{\mathrm{Fels}}[\mathrm{MPa}]$} & $\begin{array}{l}7 \text { m unter Vollstau } \\
\text { (1775 müA) }\end{array}$ & $\begin{array}{l}51 \text { m unter Vollstau } \\
\text { (1731 müA) }\end{array}$ \\
\hline & & 1. Ef [Hz] & 1. Ef [Hz] \\
\hline 40000 & \multirow{2}{*}{40000} & 1,81 & 2,31 \\
\hline 43500 & & 1,91 & 2,44 \\
\hline
\end{tabular}

\section{ABBILDUNG 7 \\ Sperre Schlegeis: Vergleich der Eigenfrequenzen \\ (angepasste Kennwerte)}

\begin{tabular}{lllll}
\multirow{2}{*}{ Mode } & \multicolumn{2}{l}{$\begin{array}{l}\text { Eigenfrequenzen [Hz] bei } \mathbf{7} \mathbf{~ m} \\
\text { unter Vollstau (1775 } \mathbf{~ m u ̈ A )}\end{array}$} & \multicolumn{2}{l}{$\begin{array}{l}\text { Eigenfrequenzen [Hz] bei } \mathbf{5 1} \mathbf{~ m} \\
\text { unter Vollstau (1731 müA) }\end{array}$} \\
\cline { 2 - 5 } & Messung & Berechnung & Messung & Berechnung \\
\hline 1 & 1,92 & 1,81 & 2,21 & 2,31 \\
\hline 2 & 2,05 & 1,86 & 2,34 & 2,49 \\
\hline 3 & 2,47 & 2,38 & 2,75 & 3,02 \\
\hline 4 & 2,93 & 2,75 & 3,23 & 3,42 \\
\hline 5 & 3,53 & 3,32 & 3,84 & 4,05 \\
\hline
\end{tabular}

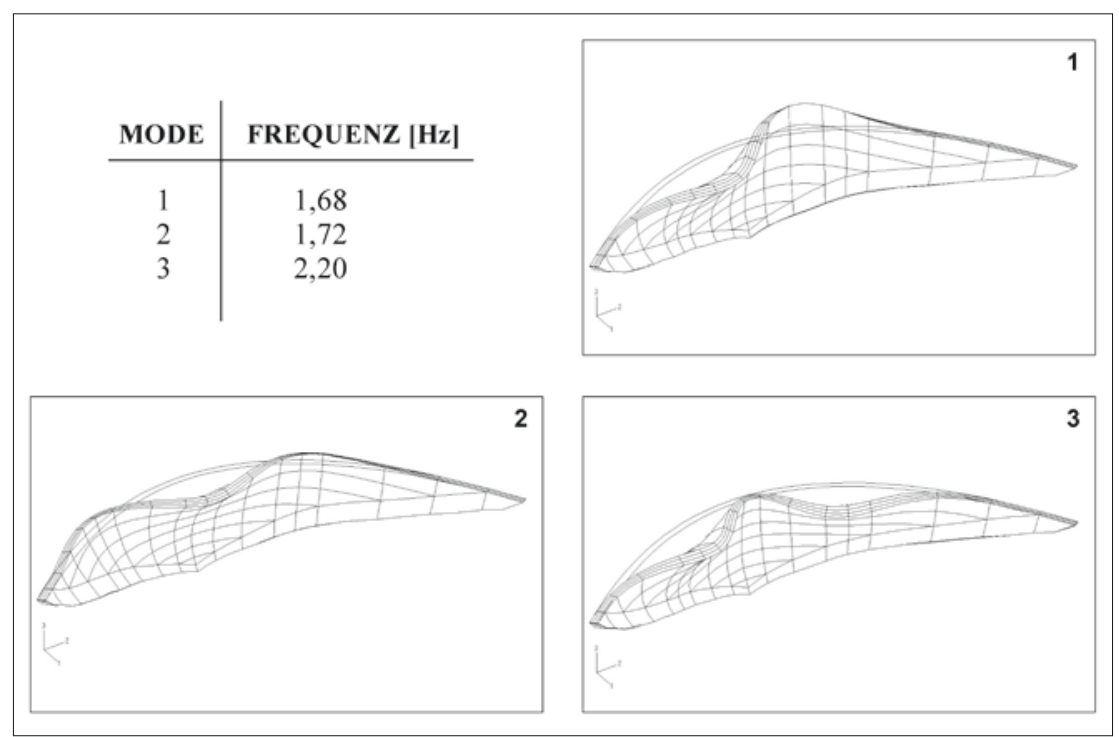

Abb. 8: Sperre Schlegeis - berechnete Eigenformen bei Vollstau. (4) höhe zeigt sich nur eine geringe Abweichung der Eigenfrequenz nach oben bzw. unten - je nach Stauspiegel. Ein Vergleich auch mit den höheren Moden ist in Abbildung 7 dargestellt.

\subsection{Eigenformen}

Mit den zuvor ermittelten Kennwerten können die nachfolgenden dynamischen Berechnungen durchgeführt werden. Als Beispiel werden hier Ergebnisse aus der Ermittlung der Eigenformen bei Vollstau in Abbildung 8 dargestellt.

\section{Zusammenfassung}

Die dargestellte Vorgehensweise zur Ermittlung von dynamischen Materialkennwerten zeigt eine komprimierte und vereinfachte Fassung von Teilen einer Erdbebenberechnung für die Sperre Schlegeis.

Das Modellverhalten ist zu identifizieren und die Kapazitäten bzw. Anwendungsgrenzen des Berechnungsmodells sind damit richtig einzuschätzen. Im Vergleich mit den Ergebnissen aus den für das Modell geeigneten Messungen lassen sich die Materialparameter durch eine Rückrechnung ermitteln und damit das numerische Modell für die Standsicherheitsberechnungen erstellen.

Korrespondenz:

er, Gerald Zenz

Technische Universität Graz, Institut für Wasserbau und Wasserwirtschaft der Technischen Universität Graz Stremayrgasse 10/l

8010Graz

http://www.hydro.tugraz.at

\section{LITERATUR}

1 Melbinger R (1998) ,,The Austrian Approach to Dam Safety: A Symbiosis of Rules and Engineering Judgement", Proc. of Int. Symp. on Dam Safety, Barcelona, 1998

2 (1995) „Standsicherheit erdbebenbeanspruchter Sperren IDNDR“, Gemeinschaftsprojekt des Bundesforschungs- und Prüfzentrums Arsenal, Abteilung Dynamik und Akustik, und des Instituts für allgemeine Mechanik/TU Wien, Zwischenbericht

3 (1991) Dams in Austria, „Schlegeis Arch Dam“, Austrian National Committee on Large Dams, pp137-140, Vienna

4 Zenz G, Obernhuber P (2002) „Erdbebenberechnung von Talsperren in Österreich", Wasserwirtschaft - Zeitschrift für Wasser und Umwelt 5 Riezinger J (1996) „Identifikation des dynamischen Verhaltens einer Talsperre bei unterschiedlichenWasserständen im Speicher", Dissertation TU Wien

6 Zenz G (2009) „Design and Analysis of Concrete Dams“", Proc. of Int. Conf. on Long Term Behaviour of Dams, Graz 